REVISTA ELEETRÉNICA CALIDAD EN LA EDUCACIÍN SUPERIOR

Instituto de Gestión de la Calidad Académica

Universidad Estatal a Distancia, Casta Rica
ISSN: I659-4703, VDL. 8(2) للالالLID-DICIEMBRE, 2017: 137-157

http://investiga.uned.ac.cr/revistas/index.php/revistacalidad

Carrea electránica: revistacalidad 国uned.ac.сг

DOI: http://dx.doi.org/10.22458/caes.v8i2.1922

\title{
PROPUESTA DE GESTIÓN EN ORIENTACIÓN EDUCATIVA PARA EL SISTEMA UNIVERSITARIO A DISTANCIA
}

\begin{abstract}
PROPOSAL OF MANAGEMENT IN EDUCATIONAL ORIENTATION FOR THE DISTANCE UNIVERSITY SYSTEM
\end{abstract}

\section{Ligia M. Arguedas-Ramírez ${ }^{1}$ larguedas@uned.ac.cr Universidad Estatal a Distancia, Costa Rica}

Volumen 8, Número 2

30 de noviembre de 2017

pp.137 - 157

Recibido: 21 de marzo de 2017

Aprobado: 8 de julio de 2017

1 MSc. Coordinadora, Programa de Orientación y Atención Psicoeducativa-POAP, Investigadora Académica, POAP-PROIFED, Universidad Estatal a Distancia-UNED, Costa Rica. Correo electrónico: larguedas@uned.ac.cr

Propuesta de Gestión en Orientación Educativa para el Sistema Universitario a Distancia Ligia M. Arguedas-Ramírez

DOI: http://dx.doi.org/10.22458/caes.v8i2.1922

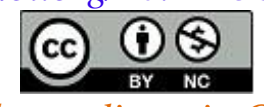

Artículo protegido por licencia Creative Commons 
REVISTA ELECTRÉNICA CALIDAD EN LA EDICACIÍN SUPERIIRR

Instituta de Gestión de la Calidad Académica

Universidad Estatal a Distancia, Costa Rica
ISSN: 1659-4703, VDL. 8(2) للالLIO-DICIEMBRE, 2017: 137-157

http://investiga.uned.ac.cr/revistas/index.php/revistacalidad

Carrec electrónica: revistacalidad国uned.ac.cए

DOI: http://dx.doi.org/10.22458/caes.v8i2.1922

\section{Resumen}

El propósito del artículo es presentar la propuesta de un Área de Investigación, Vinculación y Desarrollo Académico, con siglas AIVDA, en el ámbito de la orientación educativa, el acompañamiento y desarrollo estudiantil y los procesos de investigación del Programa de Investigación en Fundamentos de la Educación a Distancia-PROIFED, de la Universidad Estatal a Distancia (UNED), Costa Rica. La experiencia y puesta en marcha de una iniciativa de trabajo conjunto ha permitido articular dos grandes espacios en el ámbito de la gestión universitaria a distancia y el fortalecimiento académico del estudiantado. Por una parte, la Vicerrectoría de Investigación, como marco de acción académica; por otra, el desarrollo, la aplicación e insumo de resultados de investigación en los procesos de orientación educativa; como marco de acción de la orientación estudiantil. El AIVDA se integra como área del PROIFED con aporte y apoyo a los diferentes programas y servicios que ofrece la Oficina de Orientación y Desarrollo Estudiantil-OFODE a todas las y los estudiantes de la Universidad y demás instancias académicas. Los objetivos, ejes conceptuales y acciones gestoras del AIVDA se contextualizan en el campo de la investigación en educación a distancia y favorecer el desarrollo y la práctica de un modelo teórico, una red de investigación y un portafolio de proyectos académicos vinculados institucionalmente y enfocados en la población estudiantil; un área de trabajo que fundamentaría su quehacer en el enfoque de responsabilidad social universitaria y sus impactos en la formación académica, la investigación y la epistemología como referentes principales de las universidades en su ámbito de influencia.

Palabras clave: investigación; vinculación; desarrollo académico; innovación; globalización; comunidad de aprendizaje; responsabilidad social universitaria.

\section{Abstract}

The purpose of this article is to present the proposal for an Area of Research, Linkage and Academic Development, with acronyms AIVDA, in the field of educational guidance, accompaniment and student development and research processes of the Fundamental Research Program Distance Education-PROIFED, UNED, Costa Rica. The experience and implementation of an initiative of joint work has allowed to articulate two great spaces in the scope of university management at a distance and the academic strengthening of the student body. On the one hand, the Vicerrectoría de Investigación; as a framework for academic action and, on the other, the development, application and input of research results in the processes

Propuesta de Gestión en Orientación Educativa para el Sistema Universitario a Distancia Ligia M. Arguedas-Ramírez.

DOI: http://dx.doi.org/10.22458/caes.v8i2.1922

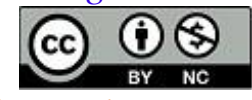

Artículo protegido por licencia Creative Commons 
REVISTA ELECTRÍNICA CALIDAD EN LA EDULACIÍN SUPPERIIRR

Instituta de Gestión de la Calidad Académica

Universidad Estatal a Distancia, Casta Rica
ISSN: 1659-4703, VQL. 8(2) للالLLIO-DICIEMBRE, 2017: 137-157

http://investiga.uned.ac.cr/revistas/index.php/revistacalidad

Correa electrónica: revistacalidad国uned.ac.cr

DOI: http://dx.doi.org/10.22458/caes.v8i2.1922

of Educational Orientation; as a framework for student orientation. The AIVDA is integrated as PROIFED Area with contribution and support to the different Programs and services offered by the Office of Orientation and Student Development-OFODE to all students of the University and other academic instances. In its structure the AIVDA: its objectives, conceptual axes and its management actions are contextualized in the field of research in foundations of distance education that favors the development and practice of - a theoretical model, a research network and a portfolio of academic projects - linked institutionally and focused on the student population. A work area that would base their work on the approach of University Social Responsibility and its impact on academic training, research and epistemology as main reference of the Universities in their sphere of influence.

Keywords: research; vinculation; academic development; innovation; globalization; learning community; university social responsibility.

\section{Introducción}

La atención y acompañamiento académico de las estudiantes y los estudiantes de la Universidad, en el marco de los servicios y procesos de orientación educativa, en sistemas de educación universitaria a distancia requieren de una decisiva formalización -regulada y normada - que permita consolidar los esfuerzos ya existentes y acceder en forma natural e integrada a una vinculación efectiva con el quehacer sustantivo de la Universidad: docencia, investigación, extensión, producción didáctica y su anclaje y proyección en los centros universitarios.

Dado lo anterior, el Programa de Orientación y Atención Psicoeducativa-POAP, de la UNED, ha desarrollado un proyecto de colaboración con la Vicerrectoría de Investigación, el Programa de Investigación en Fundamentos de la Educación a Distancia-PROIFED, particularmente, el

Propuesta de Gestión en Orientación Educativa para el Sistema Universitario a Distancia Ligia M. Arguedas-Ramírez.

DOI: http://dx.doi.org/10.22458/caes.v8i2.1922

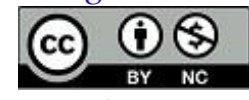

Artículo protegido por licencia Creative Commons 
REVISTA ELECTRÍNICA CALIDAD EN LA EDULACIÍN SUPPERIIRR

Instituta de Gestión de la Calidad Académica

Universidad Estatal a Distancia, Costa Rica
ISSN: 1659-4703, VQL. 8(2) للالLLIO-DICIEMBRE, 2017: 137-157

http://investiga.uned.ac.cr/revistas/index.php/revistacalidad

Carrec electrónica: revistacalidad国uned.ac.cए

DOI: http://dx.doi.org/10.22458/caes.v8i2.1922

Área de Investigación en Lenguaje, Cultura y Cognición, cuyo propósito encierra procesos y estrategias de orientación estudiantil sustentadas en investigaciones, estudios, materiales didácticos formativos que permitan unir esfuerzos en esa línea de acción. Y es el marco que da paso al propósito y planteamiento de la propuesta de un Área de Investigación, Vinculación y Desarrollo Académico, con siglas: AIVDA, que establezca y amplíe el accionar desde los fundamentos de la educación a distancia y la mirada y vivencia estudiantil.

\section{A. Criterios propuesta AIVDA}

Algunos de los razonamientos que justifican la propuesta del AIVDA, desde el contexto estudiantil de la UNED, están relacionados con cinco grandes criterios:

Propuesta de Gestión en Orientación Educativa para el Sistema Universitario a Distancia

Ligia M. Arguedas-Ramírez.

DOI: http://dx.doi.org/10.22458/caes.v8i2.1922

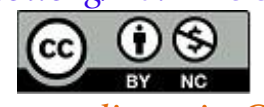

Artículo protegido por licencia Creative Commons 
Figura 1. Criterios propuesta AIVDA

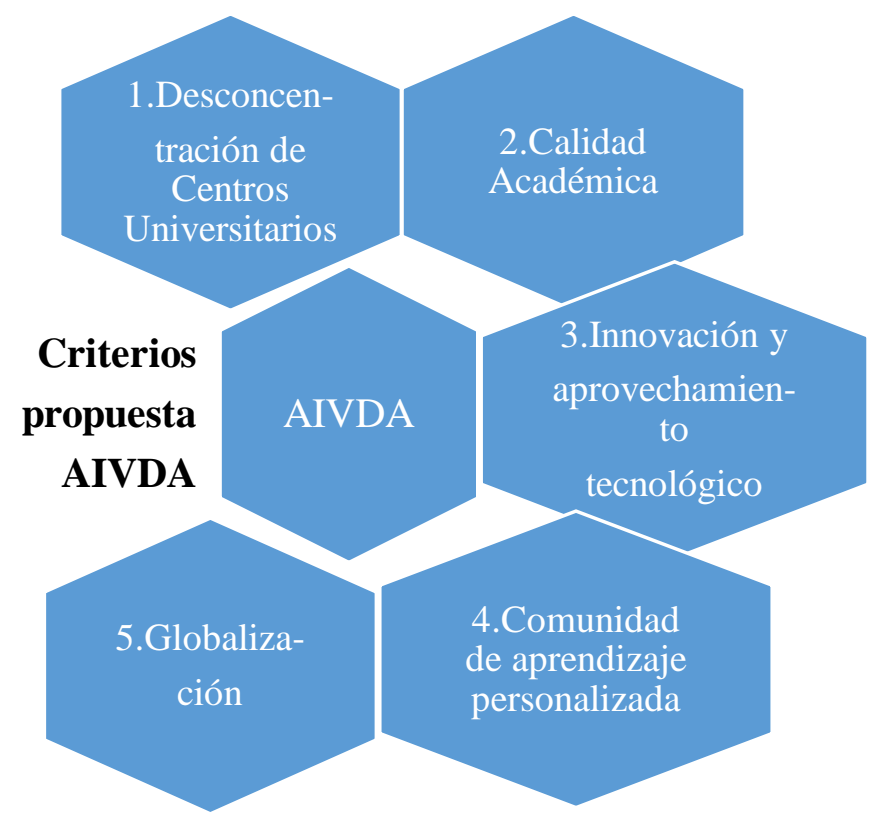

Fuente: Arguedas (2016). Proyecto POAP - PROIFED

1. Desconcentración de centros universitarios

Sustentado en el marco de las diferentes propuestas y lineamientos institucionales sobre desconcentración de los centros universitarios, el POAP evidencia la necesidad y urgencia de poder colaborar, proyectar y fortalecer oportunidades -para todas las y los estudiantes universitarios-, en servicios, proyectos, investigaciones y procesos de orientación, psicoeducativos, de acompañamiento académico y desarrollo estudiantil, que de manera desconcentrada, regional y en coordinación con otras instancias y

Propuesta de Gestión en Orientación Educativa para el Sistema Universitario a Distancia Ligia M. Arguedas-Ramírez.

DOI: http://dx.doi.org/10.22458/caes.v8i2.1922

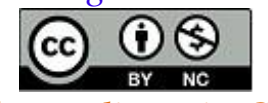

Artículo protegido por licencia Creative Commons 
REVISTA ELEETRÉNICA CALIDAD EN LA EDUCACIÍN SUPERIIRR

Instituta de Gestión de la Calidad Académica

Universidad Estatal a Distancia, Casta Rica
ISSN: 1659-4703, VQL. 8(2) للالLLIO-DICIEMBRE, 2017: 137-157

http://investiga.uned.ac.cr/revistas/index.php/revistacalidad

Coгrea electrónica: revistacalidad国uned.ac.cr

DOI: http://dx.doi.org/10.22458/caes.v8i2.1922

programas pertinentes pueda desplegar su accionar a la luz de las necesidades estudiantiles. De manera que desde la realidad y especificidad de los centros universitarios se brinde un aporte sustantivo y transversal de sus diferentes propuestas en un quehacer universitario de calidad. Por tanto, es oportuno lo planteado por la UNESCO (2015), sobre el impacto social de las universidades:

(...) en tanto referente y actor que puede (o no) promover el progreso, crear capital social, vincular a los estudiantes con la realidad exterior, hacer accesible el conocimiento a todos, etc. La universidad responsable se pregunta cómo puede acompañar el desarrollo de la sociedad y ayudar a resolver sus problemas fundamentales. (p.9)

Así, la UNED representa un aporte sustantivo para el desarrollo regional y comunitario desde sus más de treinta centros universitarios en todo el país.

\section{Calidad académica}

La calidad en el ámbito de la educación superior, según el Quinto Informe del Estado de la Educación en Costa Rica, 2015, se halla cada vez más relacionada con el desempeño universitario en cuatro aspectos clave: el seguimiento de graduados, el rendimiento educativo, la tasa de titulación y el gasto que los hogares destinan a este rubro. Por consiguiente, supone un compromiso de todas las instancias institucionales propiciar acciones afirmativas; no solo para favorecer la admisión, sino también que el estudiantado, principalmente el de menor ingreso, permanezca y se titule con una educación que sea pertinente y de relevancia para el país y el mundo.

Propuesta de Gestión en Orientación Educativa para el Sistema Universitario a Distancia Ligia M. Arguedas-Ramírez.

DOI: http://dx.doi.org/10.22458/caes.v8i2.1922

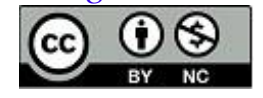

Artículo protegido por licencia Creative Commons 
REVISTA ELEETRÍNICA CALIDAD EN LA EDULACIÍN SUPPERIIRR

Instituta de Gestión de la Calidad Académica

Universidad Estatal a Distancia, Casta Rica
ISSN: 1659-4703, VDL. 8(2) للالLLIO-DICIEMBRE, 2017: 137-157

http://investiga.uned.ac.cr/revistas/index.php/revistacalidad

Coгrea electrónica: revistacalidad国uned.ac.cr

DOI: http://dx.doi.org/10.22458/caes.v8i2.1922

En el caso de la UNED, los requerimientos del modelo a distancia y el tipo de ingreso abierto en la mayoría de sus carreras, la exigencia de calidad académica implica alcanzar una correspondencia entre las mejores condiciones y experiencias de enseñanza y aprendizaje que se puedan brindar y ofertar en todos los programas de preparación profesional, por una parte, con la identificación del bagaje y la preparación académica previa de cada estudiante admitido; por otra, que en su conjunto la Universidad represente una oportunidad real de estudio y éxito para las y los estudiantes.

Es así como en los planteamientos de la UNESCO (2015), sobre el tema de "educación para todos", destacan la calidad como componente imprescindible para alcanzar el objetivo fundamental de la equidad en las instituciones educativas. Sin embargo, también puntualizan que no basta con ampliar el acceso a la educación para contribuir plenamente al desarrollo de las y los estudiantes, sino que debe darse una reciprocidad simultánea entre mejorar el acceso, en nuestro caso en el contexto universitario, y mejorar la calidad de los procesos académicos de formación profesional brindados.

\section{La innovación y el aprovechamiento tecnológico}

En la actualidad la necesidad de innovar y aprovechar la tecnología en todos los ámbitos universitarios es fundamental en los procesos educativos de forma que su uso se revierta, en los diferentes frentes y espacios, en beneficio y desarrollo del estudiantado: en los procesos de admisión a las carreras, las acciones que acompañan su permanencia y finalmente su graduación y obtención de un título que les permita crecer

e incorporarse al mundo laboral con atestados de calidad. De manera que innovar y

Propuesta de Gestión en Orientación Educativa para el Sistema Universitario a Distancia

Ligia M. Arguedas-Ramírez.

DOI: http://dx.doi.org/10.22458/caes.v8i2.1922

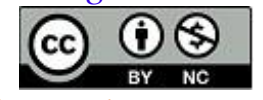

Artículo protegido por licencia Creative Commons 
REVISTA ELEETRÍNICA CALIDAD EN LA EDULACIÍN SUPPERIIRR

Instituta de Gestión de la Calidad Académica

Universidad Estatal a Distancia, Casta Rica
ISSN: 1659-4703, VDL. 8(2) للالLLIO-DICIEMBRE, 2017: 137-157

http://investiga.uned.ac.cr/revistas/index.php/revistacalidad

Coгrea electrónica: revistacalidad国uned.ac.cr

DOI: http://dx.doi.org/10.22458/caes.v8i2.1922

asociar el uso de las TIC en los servicios de orientación educativa es una tarea que se concibe con el propósito de acercar y personalizar la atención y el desarrollo académico, personal-social, vocacional y ocupacional del estudiante, mediante estrategias pedagógicas innovadoras y el uso de tecnologías virtuales, multimediales e interactivas, acordes con el sistema universitario a distancia y articulado con procesos y resultados de investigación.

\section{Comunidad de aprendizaje personalizado}

Lograr dar un salto comprometido que dirija hacia una comunidad de aprendizaje personalizado en educación a distancia. Tal como lo plantea Hernando (2015), supone una institución que "...actúa, cambia, crece y se desarrolla atenta al presente, a la investigación y a la realidad global y local (...) que descubre su identidad (...) que permite aprender para otra vida, descubrir otro mundo y crear otra narración de sí mismos", (p.10). De forma que la UNED, para cada estudiante, represente una oportunidad que potencie su aspiración de democratizar la educación superior en Costa Rica.

\section{Globalización}

Evidentemente, el fenómeno de la globalización afecta todos los ámbitos de la vida de las personas y el mundo laboral. Con mayor frecuencia, impone perfiles y competencias profesionales que tanto las universidades como el estudiantado deben atender si se quiere ser parte activa del desarrollo social y económico del país. Los perfiles suponen, en las y los estudiantes, calidades superiores de pensamiento, de aprendizaje y estudio, que les asegure una experiencia académica exitosa; mientras para la Universidad, una

Propuesta de Gestión en Orientación Educativa para el Sistema Universitario a Distancia Ligia M. Arguedas-Ramírez.

DOI: http://dx.doi.org/10.22458/caes.v8i2.1922

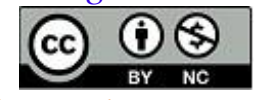


REVISTA ELEETRÍNICA CALIDAD EN LA EDULACIÍN SUPPERIIRR

Instituta de Gestión de la Calidad Académica

Universidad Estatal a Distancia, Casta Rica
ISSN: 1659-4703, VDL. 8(2) للالLLIO-DICIEMBRE, 2017: 137-157

http://investiga.uned.ac.cr/revistas/index.php/revistacalidad

Coгreo electrónica: revistacalidad国uned.ac.cг

DOI: http://dx.doi.org/10.22458/caes.v8i2.1922

planificación pedagógica, didáctica y curricular: transversal, flexible, eficaz y de aprovechamiento tecnológico, que potencie el conocimiento técnico y especializado, pero también, y de forma trascendente, los atributos personales y de habilidades sociales de cada estudiante; en un saber vivir y convivir, de compromiso, respeto, ética, dignidad: consigo mismo y los demás; en un mundo diverso y multicultural.

Lo descrito anteriormente se puede lograr en la medida que la Universidad participe y articule a todos los actores de la comunidad en una metodología a distancia -compleja y retadora-, lo cual se fundamenta muy bien en el documento de la UNESCO, (2015):

La universidad influye en la formación de los jóvenes y profesionales, su escala de valores, su manera de interpretar el mundo y de comportarse en él. Incide así mismo en la deontología profesional y orienta - de modo consciente o no- la definición de la ética profesional de cada disciplina y su rol social. La universidad responsable se pregunta por el tipo de profesionales, ciudadanos y personas que forma, y sobre la adecuada organización de la enseñanza para garantizar una formación socialmente responsable de sus estudiantes. (pág.9).

\section{B. Estructura del AIVDA}

EI AIVDA estaría circunscrito como un área del PROIFED en la Vicerrectoría de Investigación. De manera que el PROIFED sería el marco de referencia y cimiento que permitiría, como ámbito de investigación en fundamentos de educación a distancia, desarrollar: un modelo teórico, una red de investigación y un portafolio de proyectos académicos vinculados institucionalmente y enfocados en las y los estudiantes. Por otra parte, fortalecería la gestión y desarrollo de los procesos de cooperación con la OFODE, desde todos sus programas de orientación y desarrollo estudiantil, cuyo propósito en su quehacer resume Arguedas (2010, p.2):

Propuesta de Gestión en Orientación Educativa para el Sistema Universitario a Distancia Ligia M. Arguedas-Ramírez.

DOI: http://dx.doi.org/10.22458/caes.v8i2.1922

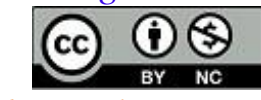

Artículo protegido por licencia Creative Commons 
Las nuevas demandas que la sociedad en general le plantea a la UNED, y a todas las instituciones de educación superior estatal, trascienden las fronteras en términos de calidad y pertinencia de la preparación universitaria de nuestros estudiantes. De ahí los aportes que como Oficina* de Orientación y Desarrollo Estudiantil podamos hacer, reflejar e impactar en la acreditación de programas de estudio, la regionalización de servicios, la equidad y el acceso a estos y, fundamentalmente, en resultados positivos en los índices de permanencia y rendimiento académico. En ese sentido la Oficina* de Orientación y Desarrollo Estudiantil, desde un modelo de educación superior a distancia, circunscribe su gestión de manera transversal, multidisciplinaria y vinculante al quehacer académico de la Universidad utilizando los recursos propios de esta metodología.

Figura 2. El AIVDA en la organización de la Vicerrectoría de Investigación

\section{EL AIVDA EN LA ORGANIZACIÓN DE LA VICERRECTORÍA DE INVESTIGACIÓN}

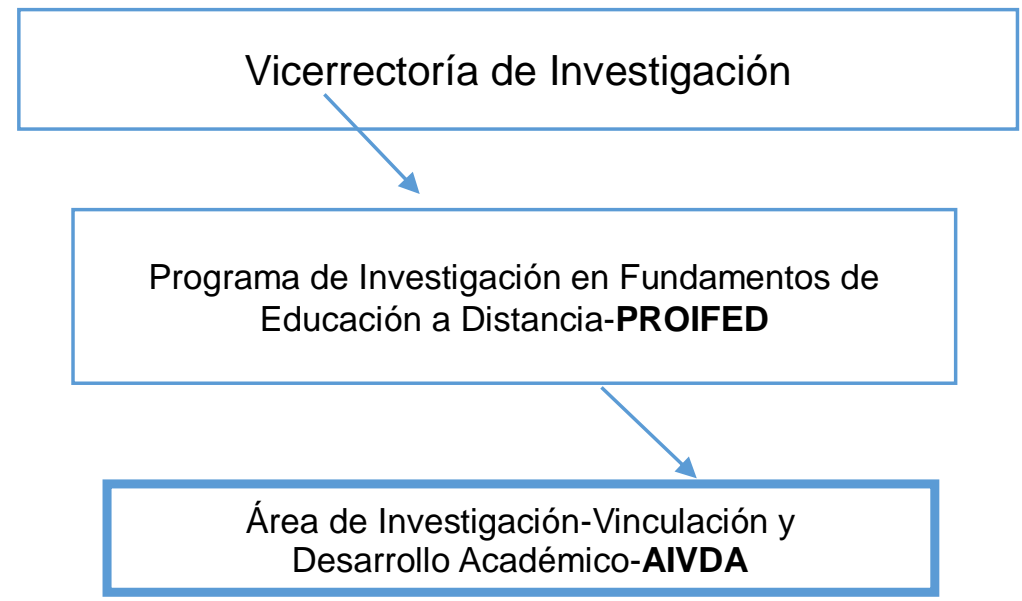

Fuente: Arguedas (2016), Proyecto POAP - PROIFED Estructura del AIVDA

\section{Propuesta de Gestión en Orientación Educativa para el Sistema Universitario a Distancia Ligia M. Arguedas-Ramírez}

DOI: http://dx.doi.org/10.22458/caes.v8i2.1922

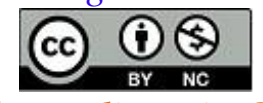


Figura 3. Estructura del AIVDA

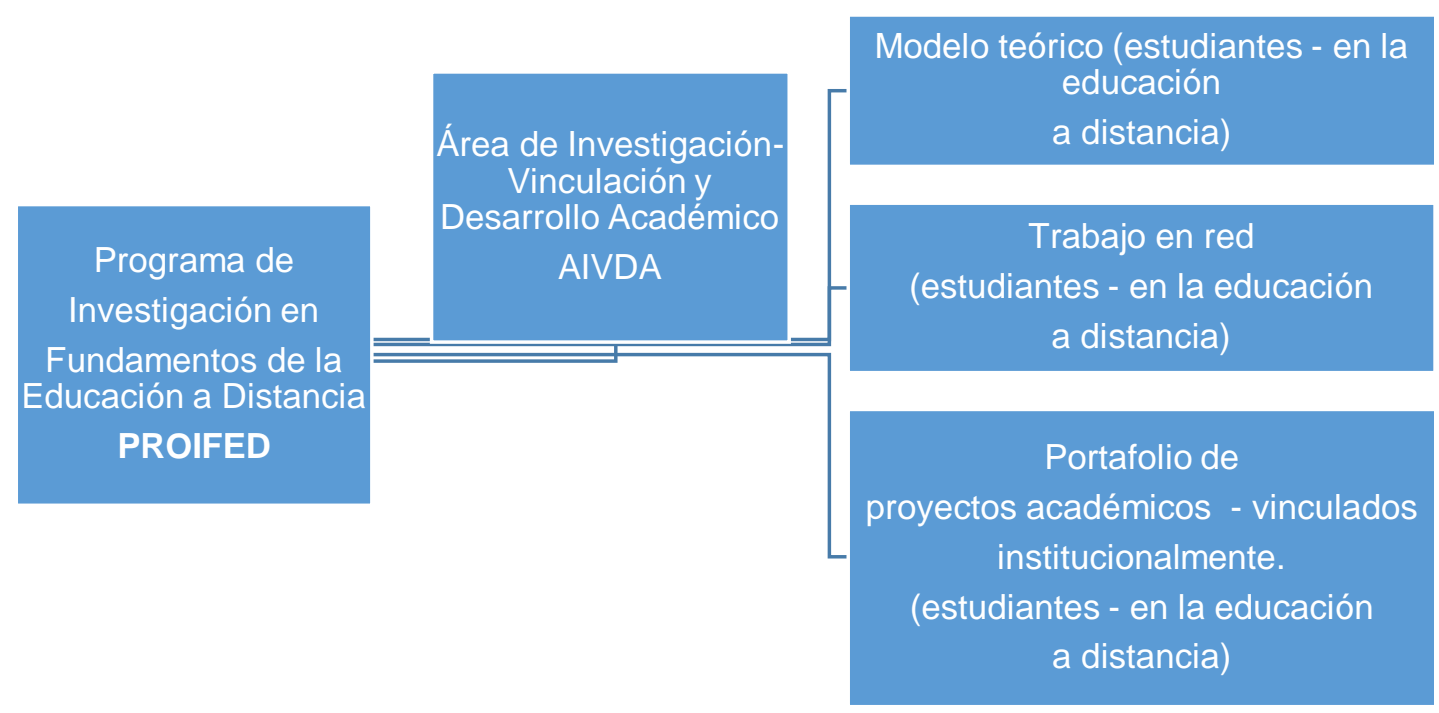

Fuente: Arguedas (2016). Proyecto POAP - PROIFED

\section{Objetivos del AIVDA}

Objetivo general:

Fundamentar desde el sistema universitario a distancia y la orientación educativa, procesos de desarrollo académico estudiantil en los ejes de investigación, didáctica y vinculación académica, que contribuyan al éxito en los estudios de las estudiantes y los estudiantes de la Universidad.

Propuesta de Gestión en Orientación Educativa para el Sistema Universitario a Distancia Ligia M. Arguedas-Ramírez

DOI: http://dx.doi.org/10.22458/caes.v8i2.1922

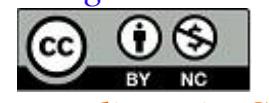

Artículo protegido por licencia Creative Commons 
REVISTA ELEETRÍNICA CALIDAD EN LA EDULACIÍN SULPERIIRR

Instituta de Gestión de la Calidad Académica

Universidad Estatal a Distancia, Casta Rica
ISSN: I659-4703, VQL. 8(2) للالLIL-DICIEMBRE, 2017: 137-157

http://investiga.uned.ac.cг/revistas/index.php/revistacalidad

Carrea electránica: revistacalidad 国uned.ac.сॅ

DOI: http://dx.doi.org/10.22458/caes.v8i2.1922

Objetivos específicos:

1. Coordinar y desarrollar estudios y proyectos de investigación educativa y estudiantil, con fundamento en la educación a distancia, que puedan ser fuente de innovación y ser capitalizados en el fortalecimiento académico de todo el estudiantado de la Universidad de manera transversal y articulada con otras instancias institucionales y externas.

2. Potenciar, en el contexto de la educación universitaria a distancia, mediante un tratamiento inclusivo, diverso y de aprovechamiento tecnológico, procesos y materiales didácticos de orientación educativa y desarrollo académico, los cuales permitan implementar proyectos y estrategias que contribuyan en la formación académica, personal, social, vocacional y ocupacional de la población estudiantil de la Universidad.

3. Propiciar vínculos académicos, con distintas instancias institucionales y externas; con propuestas y proyectos que fortalezcan la calidad de la preparación universitaria a distancia desde una perspectiva de -integración, participación, prevención, formación, aprendizaje y desarrollo estudiantil-.

\section{Ejes conceptuales del AIVDA}

- Responsabilidad social universitaria:

Lo ejes conceptuales y de acción del AIVDA están relacionados con el enfoque de responsabilidad social universitaria y con lo que Vallaeys, De la Cruz y Sasia (2009) definen como los cuatro tipos de impactos universitarios: organizacionales, sociales, cognitivos y

Propuesta de Gestión en Orientación Educativa para el Sistema Universitario a Distancia Ligia M. Arguedas-Ramírez.

DOI: http://dx.doi.org/10.22458/caes.v8i2.1922

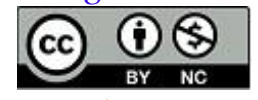

Artículo protegido por licencia Creative Commons 
REVISTA ELECTRÍNICA CALIDAD EN LA EDULACIÍN SUPPERIIRR

Instituto de Gestión de la Calidad Académica

Universidad Estatal a Distancia, Costa Rica
ISSN: I659-4703, VDL. 8(2) للالLLIO-DICIEMBRE, 2017: I37-I57

http://investiga.uned.ac.cr/revistas/index.php/revistacalidad

Carrec electrónica: revistacalidad国uned.ac.cए

DOI: http://dx.doi.org/10.22458/caes.v8i2.1922

educativos. Los dos primeros viables en cualquier tipo de institución y los dos segundos del ámbito académico, propios de instituciones educativas.

Los autores citados parten de que las universidades provocan un impacto, una "huella" social en el contexto en el que se desenvuelven, lo cual es una apreciación que, necesariamente, debe ser incorporada en el análisis institucional y hacerla formar parte de la planificación y organización universitaria como sistema: de sus instancias académicas y de los procesos y servicios de orientación estudiantil. Lo resumen de la siguiente manera: la responsabilidad social universitaria "(...) no puede ahorrarse una reflexión epistemológica acerca de los modos de (...) gestión del conocimiento que, explícita o implícitamente, se proponen en las universidades." (p.10). Por otra parte, y como aspecto esencial en los procesos de orientación educativa para el estudiantado:

(...) no se trata solo de insistir en las tres funciones sustantivas que son la docencia, la investigación y la extensión, y de confiar a ésta última la tarea de vincular a la universidad con la sociedad. Son los cuatro procesos -gestión, formación, conocimiento y participación social- los que constituyen la función social universitaria. (p.10)

En el siguiente esquema se refleja lo anterior apropiadamente:

Propuesta de Gestión en Orientación Educativa para el Sistema Universitario a Distancia Ligia M. Arguedas-Ramírez.

DOI: http://dx.doi.org/10.22458/caes.v8i2.1922

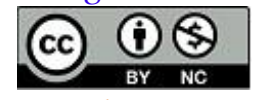

Artículo protegido por licencia Creative Commons 
REVISTA ELECTRÍNICA CALIDAD EN LA EDUCACIÍN SUPERRIDR

Instituta de Gestión de la Calidad Académica

Universidad Estatal a Distancia, Casta Rica
ISSN: I659-4703, VDL. 8(2) للالالLID-DICIEMBRE, 2017: 137-157

http://investiga.uned.ac.cr/revistas/index.php/revistacalidad

Carrea electránica: revistacalidad 国uned.ac.сг

DOI: http://dx.doi.org/10.22458/caes.v8i2.1922

Figura 4. Cuatro tipos de impactos universitarios

De acuerdo con el esquema, el eje vertical es común a cualquier tipo de organizaciones (todas generan impactos laborales, ambientales y sociales), mientras que el eje horizontal corresponde específicamente a las instituciones de aprendizaje y conocimiento (podríamos designarlo como eje académico). Analicemos brevemente cada tipo de impacto.

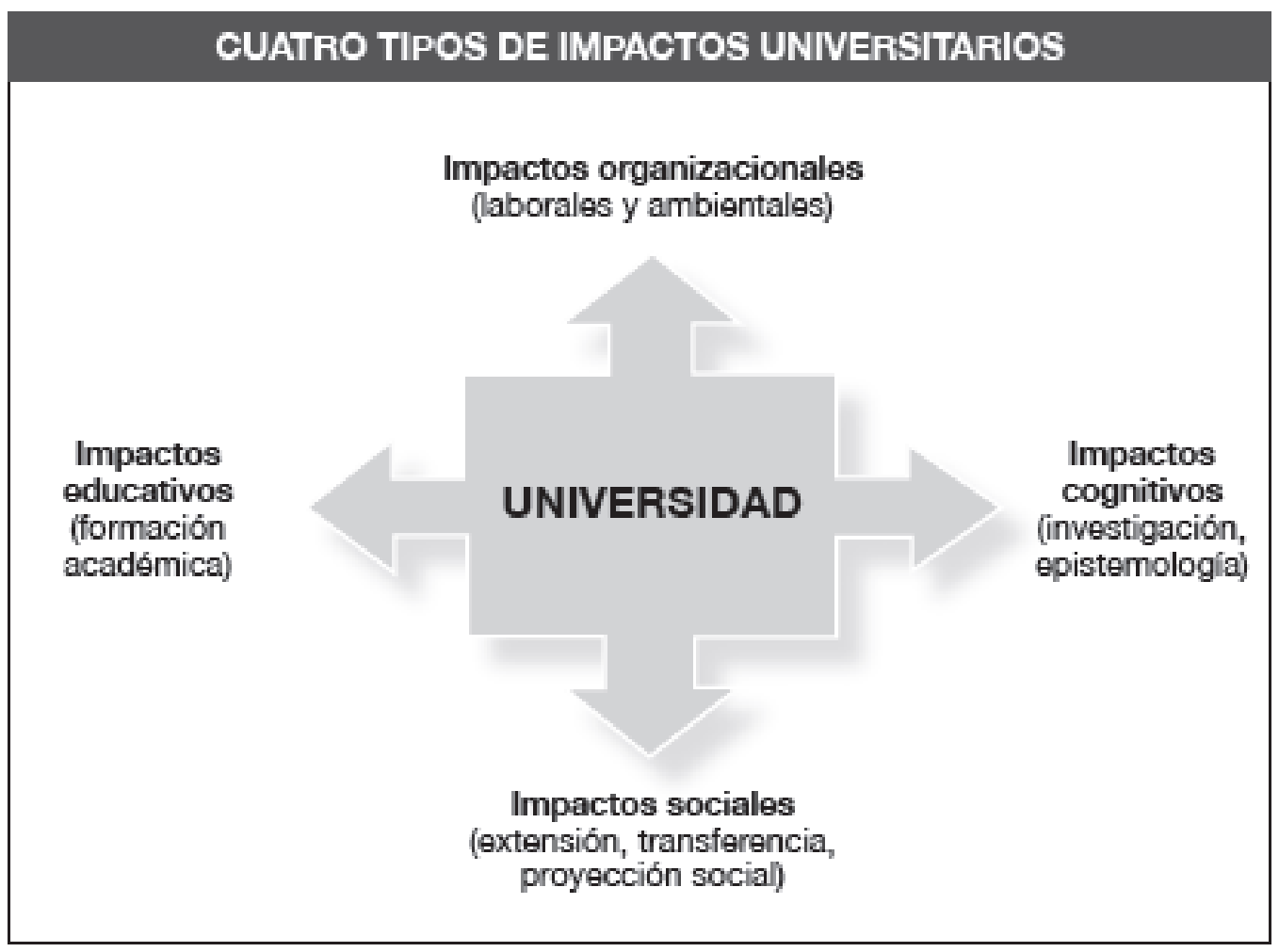

Esquema 2. La universidad y su entorno

Fuente: Vallaeys, De la Cruz y Sasia (2009, p.8).

Propuesta de Gestión en Orientación Educativa para el Sistema Universitario a Distancia Ligia M. Arguedas-Ramírez.

DOI: http://dx.doi.org/10.22458/caes.v8i2.1922

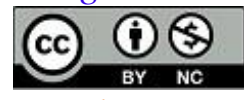

Artículo protegido por licencia Creative Commons 
Figura 5. Ejes conceptuales del AIVDA

Ejes conceptuales del AIVDA

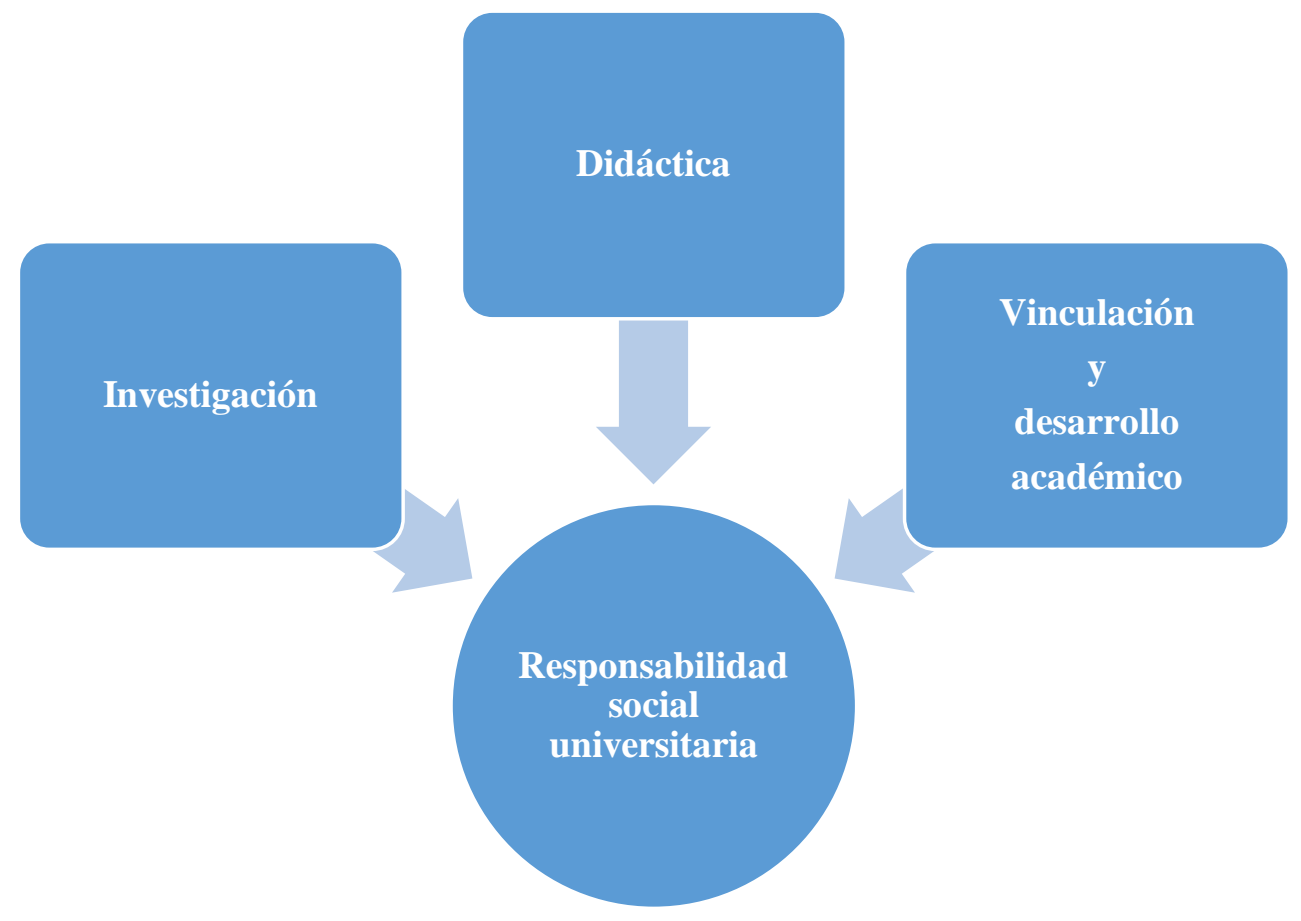

Fuente: Arguedas (2016). Proyecto POAP-PROIFED

- Investigación

La calidad y eficacia universitaria supone tanto en el ámbito académico como en el de la orientación educativa, fundamentar su quehacer en procesos de investigación, de manera que se pueda identificar y cubrir necesidades reales del estudiantado tanto en los programas de

Propuesta de Gestión en Orientación Educativa para el Sistema Universitario a Distancia Ligia M. Arguedas-Ramírez.

DOI: http://dx.doi.org/10.22458/caes.v8i2.1922

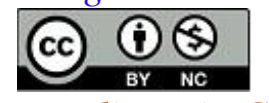

Artículo protegido por licencia Creative Commons 
REVISTA ELECTRÍNICA CALIDAD EN LA EDULACIÍN SUPPERIIRR

Instituto de Gestión de la Calidad Académica

Universidad Estatal a Distancia, Casta Rica
ISSN: I659-4703, VQL. 8(2) للالLIL-DICIEMBRE, 2017: 137-157

http://investiga.uned.ac.cr/revistas/index.php/revistacalidad

Coгrea electrónica: revistacalidad国uned.ac.cr

DOI: http://dx.doi.org/10.22458/caes.v8i2.1922

estudio como en su acompañamiento académico. Lo anterior es clave, particularmente, en los sistemas de educación a distancia, en los que su complejidad demanda establecer bases teóricas y conceptuales sólidas que sustenten los diferentes servicios y apoyos estudiantiles potenciando los principios de esta modalidad de estudio y aprendizaje: el estudio independiente, la autorregulación del aprendizaje, los procesos metacognitivos necesarios y de mediación de los aprendizajes, entre otros. La investigación educativa como lo expone Carr (2007), permite reflexionar sobre la práctica educativa, desde todos los ámbitos del proceso e implementar acciones que fortalezcan y mejoren su impacto. En el caso de los servicios de orientación educativa para las y los estudiantes, se trata de la orientación académica, el desarrollo personal, social y vocacional - ocupacional.

- Didáctica

La producción y el desarrollo de material y herramientas didácticas y educativas con experiencias tecnológicas, digitales, multimediales e innovadoras buscan fortalecer procesos formativos y de desarrollo humano del estudiantado; desde espacios no formales académicamente, y durante su preparación profesional, más allá de lo curricular y del plan de estudios. Es por demás, uno de los ejes clave y valor agregado en los sistemas de educación a distancia ya que favorecen el acompañamiento y la orientación educativa con "(...) significación personal', (Sabaté, 2006, p.19). Y se busca, según el mismo autor, que las y los estudiantes sean más conscientes de sí mismos y de su realidad.

- Vinculación y desarrollo académico

El impacto de los servicios y procesos de orientación educativa y desarrollo estudiantil requieren de una "(...) intervención sistémica, personalizada y secuenciada." (Sabaté, 2006,

Propuesta de Gestión en Orientación Educativa para el Sistema Universitario a Distancia

Ligia M. Arguedas-Ramírez.

DOI: http://dx.doi.org/10.22458/caes.v8i2.1922

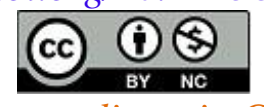

Artículo protegido por licencia Creative Commons 
REVISTA ELEETRÍNICA CALIDAD EN LA EDULACIÍN SULPERIIRR

Instituta de Gestión de la Calidad Académica

Universidad Estatal a Distancia, Casta Rica
ISSN: I659-4703, VQL. 8(2) للالLIL-DICIEMBRE, 2017: 137-157

http://investiga.uned.ac.cr/revistas/index.php/revistacalidad

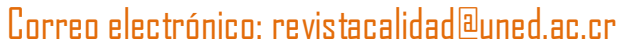

DOI: http://dx.doi.org/10.22458/caes.v8i2.1922

p.19). Lo anterior está relacionado con redes de coordinación, integración y colaboración institucional y externa que resulten en oportunidades para las y los estudiantes de todos los niveles y grados, lo cual en sistemas de educación a distancia representan un fundamento institucional relacionado con una vinculación que puede ser formal e informal, pero con propósitos claramente definidos. Por otra parte, la Universidad, desde la vida académica y estudiantil, presenta cada vez más demandas y requiere de algunos espacios de representación y participación técnica, nacional e internacional, que son de compromiso institucional, directo y preferente, ya que resguardan y fortalecen una gestión universitaria con calidad.

\section{E. Propósitos gestores del AIVDA}

$\checkmark$ Participar en el desarrollo de políticas estudiantiles en el ámbito institucional que contemplen iniciativas de articulación con las escuelas, los programas y las cátedras de la Vicerrectoría Académica, los centros de investigación y la Vicerrectoría de Investigación.

$\checkmark$ Desarrollar investigaciones y procesos educativos de seguimiento a estudiantes para favorecer su permanencia y su rendimiento académico como aporte fundamental al desarrollo estudiantil en el sistema universitario a distancia.

$\checkmark$ Desarrollar modelos teóricos en materia estudiantil en el ámbito de la educación universitaria a distancia; y desde los procesos del AIVDA.

$\checkmark$ Contextualizar la orientación como proceso educativo y de desarrollo estudiantil desde la experiencia en educación distancia.

Propuesta de Gestión en Orientación Educativa para el Sistema Universitario a Distancia Ligia M. Arguedas-Ramírez

DOI: http://dx.doi.org/10.22458/caes.v8i2.1922

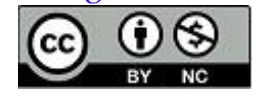

Artículo protegido por licencia Creative Commons 
$\checkmark$ Desarrollar material y procesos didácticos en orientación educativa desde un tratamiento inclusivo, diverso y de aprovechamiento tecnológico.

$\checkmark$ Generar vinculación académica con un portafolio de proyectos, estudios e investigaciones; como estrategia de orientación en el desarrollo de servicios estudiantiles, estructura institucional, apoyo a la calidad y al fortalecimiento académico.

$\checkmark$ Representación y participación técnica en comisiones y equipos de trabajo de desarrollo académico estudiantil, con otras universidades nacionales e internacionales desde el ámbito estudiantil y la Vicerrectoría de Investigación.

$\checkmark$ Fortalecer y afianzar los procesos de cooperación entre la Oficina de Orientación y Desarrollo Estudiantil-OFODE y la Vicerrectoría de Investigación: mediante procesos de investigación y formulación de servicios y materiales didácticos estudiantiles.

$\checkmark$ Desarrollar proyectos de orientación y admisión para estudiantes, en conjunto con la Escuela de Educación y su Centro de Investigación.

$\checkmark$ Desarrollar proyectos de contextualización laboral de los programas de Estudio de la UNED, vinculándolos al Observatorio Laboral de Profesiones (Consejo Nacional de Rectores-CONARE).

$\checkmark$ Mantener la representación técnica y profesional en comisiones y equipos de trabajo en el ámbito institucional en temas acordes al AIVDA, el PROIFED y la Vicerrectoría de Investigación.

Propuesta de Gestión en Orientación Educativa para el Sistema Universitario a Distancia Ligia M. Arguedas-Ramírez.

DOI: http://dx.doi.org/10.22458/caes.v8i2.1922

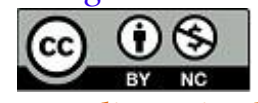

Artículo protegido por licencia Creative Commons 
REVISTA ELEETRÍNICA CALIDAD EN LA EDULACIÍN SUPPERIIRR

Instituta de Gestión de la Calidad Académica

Universidad Estatal a Distancia, Casta Rica
ISSN: 1659-4703, VQL. 8(2) للالLIO-DICIEMBRE, 2017: 137-157

http://investiga.uned.ac.cr/revistas/index.php/revistacalidad

Coгrea electrónica: revistacalidad国uned.ac.cr

DOI: http://dx.doi.org/10.22458/caes.v8i2.1922

\section{F. Reflexiones finales}

A modo de observaciones finales se destacan los siguientes aspectos:

$\checkmark$ Este tipo de iniciativas y el desarrollo de un Área como el AIVDA es una propuesta de impacto institucional. En el ámbito estudiantil pueden surgir proyectos e investigaciones que estimulen el crecimiento personal y el éxito académico acorde con los tiempos que corren y la proyección universitaria.

$\checkmark \quad$ La calidad académica debe ser una constante en todo el quehacer institucional y eje central en la responsabilidad social universitaria. Los procesos de investigación educativa no solo deben acuerpar aspectos instrumentales o técnicos en la docencia y en el acompañamiento al estudiantado, " (...) sino la recuperación de marcos teóricos, presupuestos y posiciones valorativas que enriquecen la práctica educativa. " (p.16, Méndez y Méndez, 2007)

$\checkmark$ Enfatizar que en materia estudiantil es necesario dar sostenibilidad, continuidad y proyección al quehacer de la Vicerrectoría de Investigación y al Programa de Investigación en Fundamentos de la Educación a Distancia-PROIFED, con el fin de que se apoye y fortalezca una práctica académica de calidad y el crecimiento, la vinculación y el desarrollo conjunto: las escuelas y los centros universitarios de todo el país.

$\checkmark$ Finalmente, la importancia de ampliar, sustantivamente y como apuesta deliberada, los alcances de la Vicerrectoría de Investigación y el PROIFED en la UNED al lograr potenciar y desarrollar un modelo teórico, una red de investigación y un portafolio de proyectos académicos, vinculados institucionalmente y enfocados en las y los estudiantes en sistemas de educación universitaria a distancia.

Propuesta de Gestión en Orientación Educativa para el Sistema Universitario a Distancia Ligia M. Arguedas-Ramírez.

DOI: http://dx.doi.org/10.22458/caes.v8i2.1922

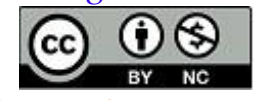

Artículo protegido por licencia Creative Commons 
REVISTA ELEETRÉNICA CALIDAD EN LA EDUCACIÍN SUPERIIRR

Instituta de Gestión de la Calidad Académica

Universidad Estatal a Distancia, Costa Rica
ISSN: I659-4703, VDL. 8(2) للالLLIO-DICIEMBRE, 2017: I37-I57

http://investiga.uned.ac.cг/revistas/index.php/revistacalidad

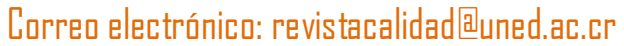

DOI: http://dx.doi.org/10.22458/caes.v8i2.1922

\section{Referencias}

Arguedas, L. (2016). Criterios Propuesta del AIVDA. Proyecto POAP - PROIFED, UNED. CR.

Arguedas, L. (2016) El AIVDA en la organización de la Vicerrectoría de Investigación. Proyecto POAP - PROIFED, UNED. CR.

Arguedas, L. (2016). Estructura del AIVDA. Proyecto POAP- PROIFED, UNED. CR.

Arguedas, L. (2016). Ejes conceptuales del AIVDA. Proyecto POAP-PROIFED, UNED. CR.

Arguedas, L. (2015). Propuesta de coordinación de servicios de orientación educativa: fortaleciendo la calidad en educación a distancia. Revista Innovaciones Educativas. Año XVII, 22, 53-64.

Arguedas, L. (2010). Propuesta de estructura área de orientación y desarrollo estudiantil. Costa Rica: UNED.

Carr, W. (Coord.) Méndez, A. y Méndez, S. (2007). El docente investigador en educación. Textos de Wilfred Car. Universidad de Ciencias y Artes de Chiapas, p.16.

Gallego, R. y Riart, J. (2006). La tutoría y la Orientación en el siglo XXI: Nuevas Propuestas. Sabaté, J. Capítulo 3: Conceptos y Agentes de la Orientación: estructura tríadica. Primera Edición, Octaedro, Barcelona, p.19.

Hernando, A. (2015). VIAJE A LA ESCUELA DEL SIGLO XXI. Así trabajan los colegios más innovadores del mundo. Editorial Fundación Telefónica, Madrid, España, p.10.

Organización de las Naciones Unidas para la Educación, la Ciencia y la Cultura. (2015). La Educación para Todos, 2000-2015: Logros y Desafíos. UNESCO. 1 ed. Francia, p.9.

Programa Estado en Desarrollo Humano Sostenible (Costa Rica). Quinto Informe Estado de la Educación/ PEN-San José, CR.

Propuesta de Gestión en Orientación Educativa para el Sistema Universitario a Distancia Ligia M. Arguedas-Ramírez.

DOI: http://dx.doi.org/10.22458/caes.v8i2.1922

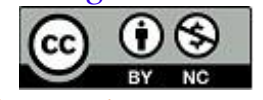

Artículo protegido por licencia Creative Commons 
REVISTA ELEETRÉNICA CALIDAD EN LA EDUCACIÍN SUPERIOR

Instituta de Gestión de la Calidad Académica

Universidad Estatal a Distancia, Casta Rica
ISSN: 1659-4703, VDL. 8(2) لالالLID-DICIEMBRE, 2017: 137-157

http://investiga.uned.ac.cr/revistas/index.php/revistacalidad

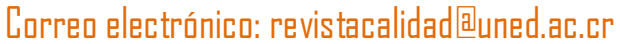

DOI: http://dx.doi.org/10.22458/caes.v8i2.1922

Vallaeys, F., De la Cruz, C. y Sasia, P. (2009). Responsabilidad Social Universitaria. Manual de primeros pasos. Mc. Graw Hill, 1 Ed. Interamericana. Figura 4. Cuatro tipos de impactos universitarios, p.8/10.

Propuesta de Gestión en Orientación Educativa para el Sistema Universitario a Distancia Ligia M. Arguedas-Ramírez

DOI: http://dx.doi.org/10.22458/caes.v8i2.1922

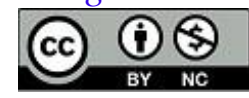

Artículo protegido por licencia Creative Commons 